\title{
Topological phase transition induced by magnetic proximity effect in two dimensions
}

\author{
Yijie Zeng ${ }^{1}$, Luyang Wang ${ }^{1, *}$, Song $\mathbf{L i}^{2}$, Chunshan $\mathrm{He}^{1}$, \\ Dingyong Zhong ${ }^{1}$ and Dao-Xin Yao ${ }^{1, * *}$ \\ ${ }^{1}$ State Key Laboratory of Optoelectronic Materials and Technologies, School of \\ Physics, Sun Yat-sen University, Guangzhou 510275, China \\ 2 Department of Mechanical and Biomedical Engineering, City Unversity of Hong \\ Kong, Kowloon, China \\ E-mail: *wangluyang730@gmail.com \\ ** yaodaox@mail.sysu.edu.cn
}

April 2019

\begin{abstract}
We study the magnetic proximity effect on a two-dimensional topological insulator in a $\mathrm{CrI}_{3} / \mathrm{SnI}_{3} / \mathrm{CrI}_{3}$ trilayer structure. From first-principles calculations, the $\mathrm{BiI}_{3}$-type $\mathrm{SnI}_{3}$ monolayer without spin-orbit coupling has Dirac cones at the corners of the hexagonal Brillouin zone. With spin-orbit coupling turned on, it becomes a topological insulator, as revealed by a non-vanishing $Z_{2}$ invariant and an effective model from symmetry considerations. Without spin-orbit coupling, the Dirac points are protected if the $\mathrm{CrI}_{3}$ layers are stacked ferromagnetically, and are gapped if the $\mathrm{CrI}_{3}$ layers are stacked antiferromagnetically, which can be explained by the irreducible representations of the magnetic space groups $C_{3 i}^{1}$ and $C_{3 i}^{1}\left(C_{3}^{1}\right)$, corresponding to ferromagnetic and antiferromagnetic stacking, respectively. By analyzing the effective model including the perturbations, we find that the competition between the magnetic proximity effect and spin-orbit coupling leads to a topological phase transition between a trivial insulator and a topological insulator.
\end{abstract}

Keywords: 2D topological insulator, magnetic proximity effect, topological phase transition, $\mathrm{CrI}_{3} / \mathrm{SnI}_{3} / \mathrm{CrI}_{3}$ heterostructure

\section{Introduction}

Topological insulators (TIs) are time-reversal invariant systems with a non-zero $Z_{2}$ index[1, 2]. They are extraordinary in that a gapless, conducting surface (edge) state exists while the bulk is insulating. The necessary condition for TIs to occur is band inversion induced by spin-orbit coupling (SOC). The first model for two-dimensional (2D) TIs is graphene[3], and later confirmed in $\mathrm{HgTe} / \mathrm{CdTe}$ heterostructure [4, [5]. To detect the edge state a sizeable bulk band gap is necessary. In the search of new 2D TIs candidate materials, two kinds of efforts are made, one is to increase the 
SOC in graphene, by forming heterostructure with materials with large SOC [6, 7, 8]. The other is to predict other TIs with large gap by first-principles calculation, such as germanene[9], tin film[10], bismuth bilayer[11] and distorted $1 T^{\prime}$ transition-metal dichalcogenide $\mathrm{MX}_{2}(\mathrm{M}=\mathrm{Mo}, \mathrm{W}$ and $\mathrm{X}=\mathrm{S}, \mathrm{Se})[12]$.

In 2D TIs, the edge state causes quantum spin Hall effect (QSHE), which is the analogue of quantum Hall effect. When there is spontaneous magnetization, the exchange interaction between the conduction electron in the edge state and the magnetic moment will gap the edge state[13], leading to quantum anomalous Hall effect (QAHE) [14, 15]. The spontaneous magnetization can be introduced by doping magnetic transition metals into the TIs[16, 17]. However, the random distribution of dopant can greatly affects the gap. Another way is to use the magnetic proximity effect by forming heterostructure with ferromagnetic insulators [18, 19] while a well-matched heterostructure is crucial.

Here we concentrate on the second way of introducing magnetism into a TI, by constructing a model $\mathrm{CrI}_{3} / \mathrm{SnI}_{3} / \mathrm{CrI}_{3}$ trilayer heterostructure. $\mathrm{CrI}_{3}$ is a layered ferromagnet with Curie temperature of $61 \mathrm{~K}[20$. In the monolayer it is Ising ferromagnet with magnetic moment perpendicular to the layer, while bilayer $\mathrm{CrI}_{3}$ shows antiferromagnetic ground state[21, 22]. By forming heterostructure with $\mathrm{SnI}_{3}$, which is shown to be a TI, it is found that ferromagnetic alignment between $\mathrm{CrI}_{3}$ layers preserves the Dirac point of $\mathrm{SnI}_{3}$, while antiferromagnetic alignment opens a gap of about $16 \mathrm{meV}$. Furthermore, ferromagnetic alignment introduces weak Van Vleck paramagnetism in $\mathrm{SnI}_{3}$, and turning it into a magnetic TI. An effective Hamiltonian including both SOC and antiferromagnetism is established, by including spin into the Haldane model[23]. In such a system phase transition between a trivial antiferromagnetic insulator and TI can occur, depending on the relative strengths of SOC and antiferromagnetism. Our findings may provide some insight to the spin-orbit torque effect[24] at the ferromagnet/TI interface [25, 26, 27], and the resulting current-induced magnetization switching by magnetic proximity effect. The electronic structure of $\mathrm{SnI}_{3}$ monolayer is discussed in Section 2, followed by the magnetic proximity effect in $\mathrm{CrI}_{3} / \mathrm{SnI}_{3} / \mathrm{CrI}_{3}$ trilayer structure. A brief conclusion is given in Section 4 .

\section{Structure and electronic properties of $\mathrm{SnI}_{3}$ monolayer}

\subsection{Results from first-principles calculations}

The bulk $\mathrm{SnI}_{3}$ is in rhombohedral $\mathrm{BiI}_{3}$ structure[28, [29, 30] (space group $R \overline{3}, C_{3 i}^{2}$, No.148), the unit cell of which can be treated in two ways. One is using rhombohedral axes, where the three basis vectors have the same length and the same angles between each other. The other is using hexagonal axes, where the basis vectors $\vec{a}, \vec{c}$ are the same as those of hexagonal crystals. Here we use the second since it is convenient to reveal the layer structure of $\mathrm{BiI}_{3}$. As a result, each unit cell contains three layers of $\mathrm{BiI}_{3}$, connected by weak van de Waals interaction, and each layer of $\mathrm{BiI}_{3}$ is composed 


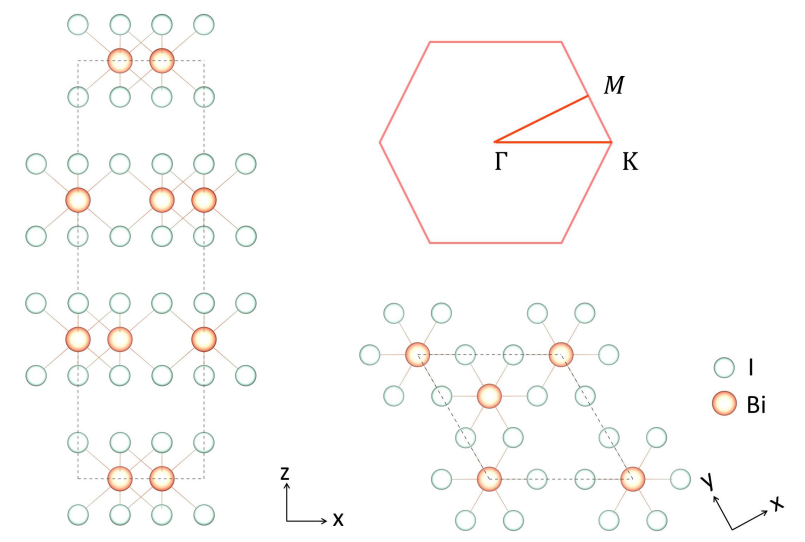

Figure 1. The unit cell of $\mathrm{BiI}_{3}$ structure seen on (12̄0) (Left), (001) (Right bottom) surface, and the Brillouin zone for the monolayer form (Right up).
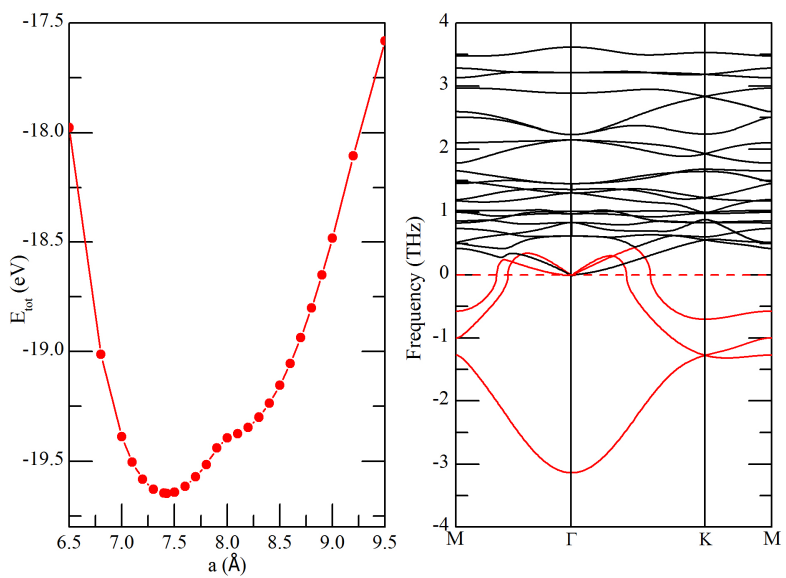

Figure 2. (Left) The total energy without SOC versus lattice constant for monolayer $\mathrm{SnI}_{3}$. (Right) Phonon dispersion of monolayer $\mathrm{SnI}_{3}$, the red curves correspond to imaginary modes.

of three atomic layers - two iodine layers and one tin layer in between, see Figure 1. There is a displacement of $(\vec{a}+\vec{b}) / 3$ between neighboring layers, where $\vec{a}$ and $\vec{b}$ are basis vectors of the unit cell.

Compared with the bulk, which has the $C_{3 i}$ point group symmetry, the monolayer has an additional twofold rotation symmetry perpendicular to $\vec{c}$, and hence belongs to the $D_{3 d}$ point group. In the early stage of our analysis, the $\mathrm{SnI}_{3}$ monolayer was supposed to be grown on $\operatorname{Ag}(111)$ surface, leading to a lattice constant of $8.7 \AA$.

To see whether strain exists at this lattice constant, we have calculated the total energies under different lattice constants, as shown in Figure 2, The lattice constant with lowest energy is $7.43 \AA$. Thus a tensile strain about $17 \%$ would exist in this monolayer if it were grown on $\operatorname{Ag}(111)$ surface. Furthermore, the phonon dispersion calculated at $8.7 \AA$ shows an imaginary optical and two imaginary acoustic phonon branches (Figure 2), indicating the monolayer might be unstable at low temperature. Hereafter we will not discuss the stability issue but pay attention to its electronic properties. The band 

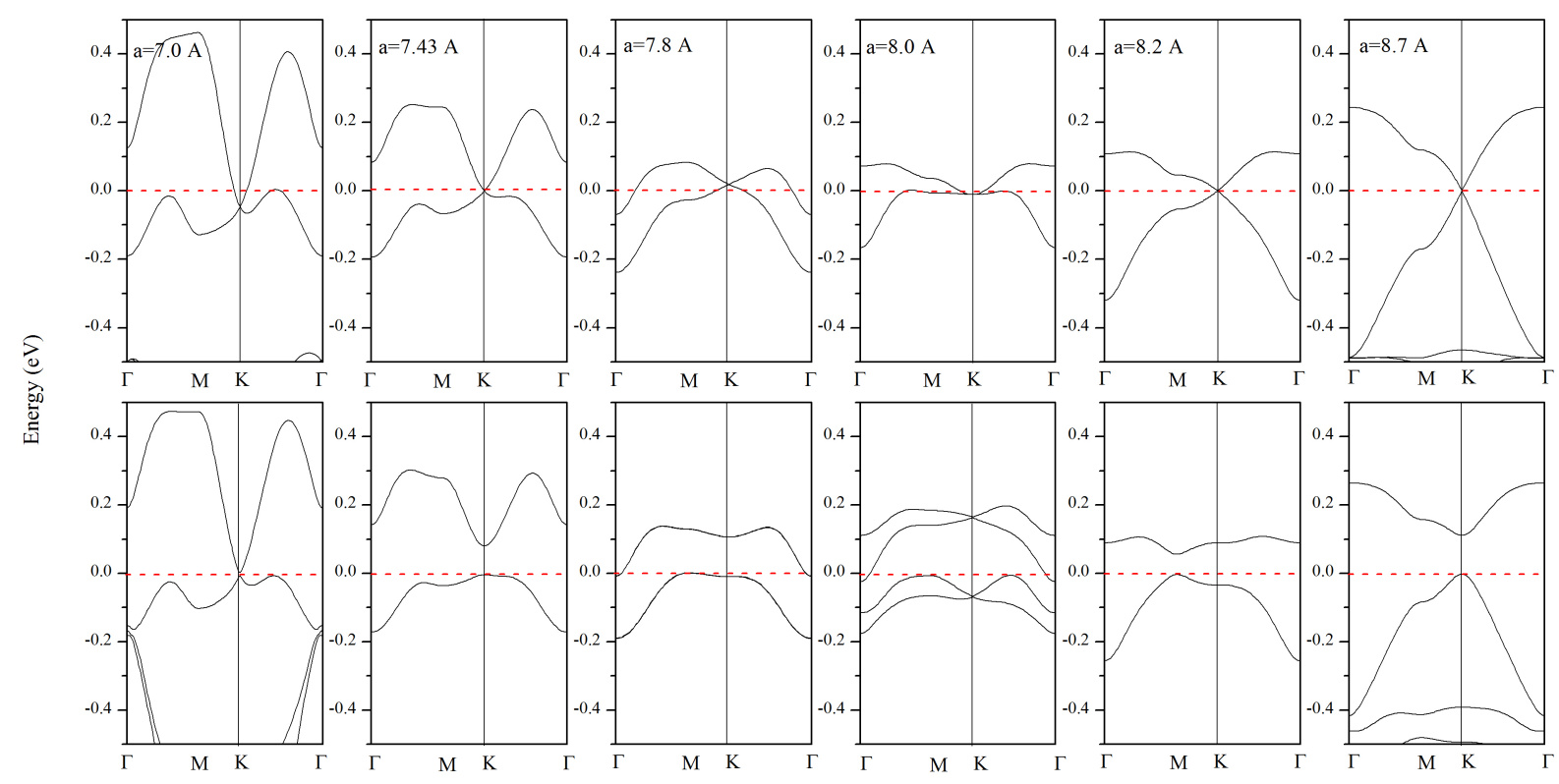

Figure 3. The band structure of the $\mathrm{SnI}_{3}$ monolayer without (Up) and with (Bottom) SOC, at different lattice constants. Only the two bands around the Fermi energy are shown.

structures without and with $\mathrm{SOC}$ of the $\mathrm{SnI}_{3}$ monolayer at selected lattice constants are shown in Figure 3. Two bands, which come from the I $p$ and Sn $s$ states, appear near the Fermi energy $\left(E_{F}\right)$, as shown in Figure 4. Without SOC, the two bands touch at $K$, realizing a two dimensional representation of the group of the wave vector at $K\left(C_{3 v}\right)$. Especially, at lattice constant of $7.43 \AA, 8.2 \AA$ and $8.7 \AA$, the energy dispersion near $K$ is linear, and $E_{F}$ locates exactly at the touching point, leading to a vanishing density of state (DOS). After including the SOC effect, which is supposed to be large due to the presence of the heavy iodine[31], a gap of $0.11 \mathrm{eV}$ opens in the Brillouin zone. Due to time-reversal symmetry and inversion symmetry, the bands are doubly degenerate at any $k$ even with SOC[32]. (There is an anomaly at $8.0 \AA$, in which case magnetism appears after including SOC, and the double degeneracy is broken. This spontaneous magnetization causes the inflection in the $E-a$ curve near $8.0 \AA$, as shown in Figure 2 (a).) At other lattice constants, there is a finite DOS at $E_{F}$ and the system is a compensated semimetal.

\subsection{Effective model}

The gaplessness without SOC and the SOC induced gap also appear in graphene 33, 34, 35], which is a semimetal without SOC and a 2D TI once the intrinsic SOC is taken into account. This leads us to ask whether the $\mathrm{SnI}_{3}$ monolayer is a $2 \mathrm{D}$ TI. The calculated $Z_{2}$ invariant is 1 (see appendix), indicating the system is a TI. Here we develop the effective theory near the degenerate point. The generic Hamiltonian near $K$ to the first 


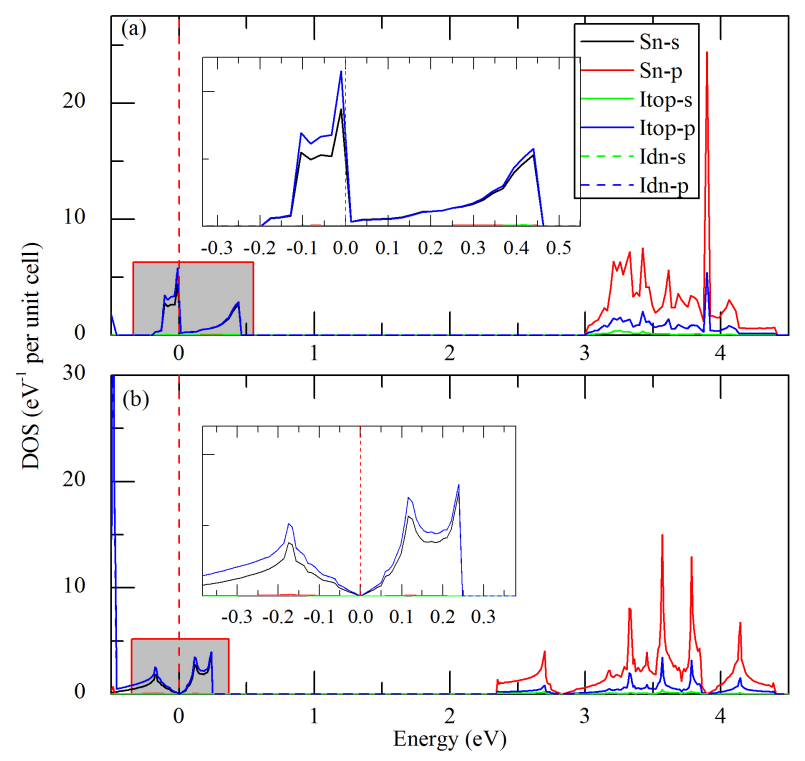

Figure 4. Density of states for $\mathrm{SnI}_{3}$ monolayer at lattice constant of (a) $7.0 \AA$ and (b) $8.7 \AA$. The insets are the enlarged region around the Fermi energy.

order of $\vec{k}$ can be written as

$$
H_{\vec{K}}(\vec{k})=c_{i} k_{i}+\vec{R}(\vec{k}) \cdot \vec{\sigma}
$$

where $\vec{R}(\vec{k})$ is a vector with each component linear in $k_{i}$, and $\sigma_{i}$ 's are the Pauli matrices of a pseudospin associated with the two bands near the Dirac point $K$. The energy spectrum is a Dirac cone. Due to the $C_{3 v}$ symmetry, the Dirac cone must be isotropic, so we can write $H_{\vec{K}}(\vec{k})=v_{F} \vec{k} \cdot \vec{\sigma}$. Here $v_{F}$ is the Fermi velocity, which is about $1.47 \times 10^{5}$ $\mathrm{m} / \mathrm{s}$ at lattice constant of $8.7 \AA$, deduced from the band structure. The magnitude of Fermi velocity is of interest for creation of bound states [36]. Time reversal requires that $H_{-\vec{K}}(\vec{k})=H_{\vec{K}}^{*}(-\vec{k})=-v_{F} \vec{k} \cdot \vec{\sigma}^{*}$. The full Hamiltonian that describes the low energy states near both $\vec{K}$ and $-\vec{K}$ is

$$
H(\vec{k})=\left(\begin{array}{cc}
H_{\vec{K}}(\vec{k}) & 0 \\
0 & H_{-\vec{K}}(\vec{k})
\end{array}\right)=v_{F}\left(k_{x} \sigma_{x} \tau_{z}+k_{y} \sigma_{y}\right)
$$

which satisfies the time reversal symmetry $T H(\vec{k}) T^{-1}=H(-\vec{k})$. Here $T=i \tau_{x} s_{y} K$ is the time reversal operation including spin, with $\tau_{i}$ 's being the Pauli matrices for the valley degree of freedom and $s_{i}$ 's the Pauli matrices for spin. Since the system also has inversion symmetry, that is, $P H(\vec{k}) P=H(-\vec{k})$, we can deduce the matrix for the inversion operator $P=\sigma_{x} \tau_{x}$ with awareness of that inversion exchanges $\vec{K}$ and $-\vec{K}$, and does not affect the spin. The SOC term compatible with both time reversal and inversion symmetry, as well as the roational symmetry of the lattice is $H_{S O}=\lambda \sigma_{z} \tau_{z} s_{z}[3$. The Rashba SOC is prohibited by the inversion symmetry. The chiral symmetry is broken with the addition of the SOC term, which is intrinsically linked to topological phases. The Hamiltonian is the same as the effective Hamiltonian of Kane-Mele model[1] without the Rashba term, so we expect the system is a $Z_{2}$ TI in $2 \mathrm{D}$. 


\section{Magnetic proximity effect in $\mathrm{CrI}_{3} / \mathrm{SnI}_{3} / \mathrm{CrI}_{3}$ trilayer structure}

\subsection{Results from first-principles calculations}

Having confirmed the $\mathrm{SnI}_{3}$ monolayer is a TI, we then construct a $\mathrm{CrI}_{3} / \mathrm{SnI}_{3} / \mathrm{CrI}_{3}$ trilayer structure, in the same stacking sequence as in bulk $\mathrm{BiI}_{3}$, to study the magnetic proximity effect on the TI. This newly designed heterostructure belongs to space group $P \overline{3}\left(C_{3 i}^{1}\right.$, No.147). The lattice constant is chosen to be $7.43 \AA$, under which the $\mathrm{CrI}_{3}$ monolayer suffers a tensile strain about $8 \%$. However, the ferromagnetic ordering is still the ground state under this tensile strain[37]. Therefore, the Cr magnetic moments in each $\mathrm{CrI}_{3}$ layer are aligned ferromagnetically, perpendicular to the layer, while both ferromagnetic and antiferromagnetic alignment between the $\mathrm{CrI}_{3}$ layers are considered. Several experimental works on the monolayer-substrate heterostructure have shown large lattice mismatch might exist for $2 \mathrm{D}$ materials. $\mathrm{CrI}_{3} / \mathrm{WSe}_{2}$ heterostructure has been fabricated to study the valley splitting of $\mathrm{WSe}_{2}$ due to magnetical exchange field of $\mathrm{CrI}_{3}$ [38], where the lattice mismatch is $5.4 \%[39]$. Another example is monolayer FeSe deposited on $\mathrm{SrTiO}_{3}(110)$ plane, where one of in-plane lattice constants is reduced by 6\%[40]. Earlier work on $\mathrm{MoS}_{2}$ even demonstrates strain as large as 11\%[41]. Moreover, the Dirac point of monolayer $\mathrm{SnI}_{3}$ exists in the whole range of lattice constants we studied, as a result of the $D_{3 d}$ symmetry. Thus we expect the lattice constant chosen for the heterostructure is reasonable and would not affect the main conclusion presented in the following.

The interplay between the magnetism in $\mathrm{CrI}_{3}$ and the topological band structure in $\mathrm{SnI}_{3}$ can be viewed from two perspectives - the effect of magnetism on the band topology, and the effect of the large SOC on the magnetism. First let's look at the influence of magnetism on the band structure. Due to the weak van de Waals interaction, the two bands near $E_{F}$ are still from $\mathrm{SnI}_{3}$. However, this weak interaction has some influence on the two bands, compared with the monolayer case (see Figure 3), that the bottom band raises higher along $K \Gamma$ than the Dirac point, leading to a compensated semimetal. The ferromagnetic spin-up band structure is different from the spin-down band structure due to the exchange potentials seen by spin-up and spin-down electrons are different [42, manifesting in that several bands appear between $-0.4 \mathrm{eV}$ and $-0.6 \mathrm{eV}$ for spin-up electrons. The antiferromagnetic spin-up and spin-down band structures are the same, due to the equivalence of spin-up and spin-down electrons. The striking difference between the ferromagnetic and antiferromagnetic band structures is that, the Dirac point at $K$ is preserved under ferromagnetic alignment, while a gap (about $16 \mathrm{meV}$ ) is opened under antiferromagnetic alignment, see Figure 5 (b) and (d). Furthermore, under antiferromagnetic alignment, only the double degeneracy at $K$ is split, while the double degeneracy at $\Gamma$ is preserved.

This peculiar behavior can be understood as follows. The magnetic space groups of the heterostructure under ferromagnetic and antiferromagnetic alignments are $C_{3 i}^{1}$ and $C_{3 i}^{1}\left(C_{3}^{1}\right)$, respectively 43 , 44]. In the former case the time-reversal symmetry $T$ is absent, while in the latter $T$ exists in combination with $I, S_{6}^{+}$and $S_{6}^{-}$. By inspecting 

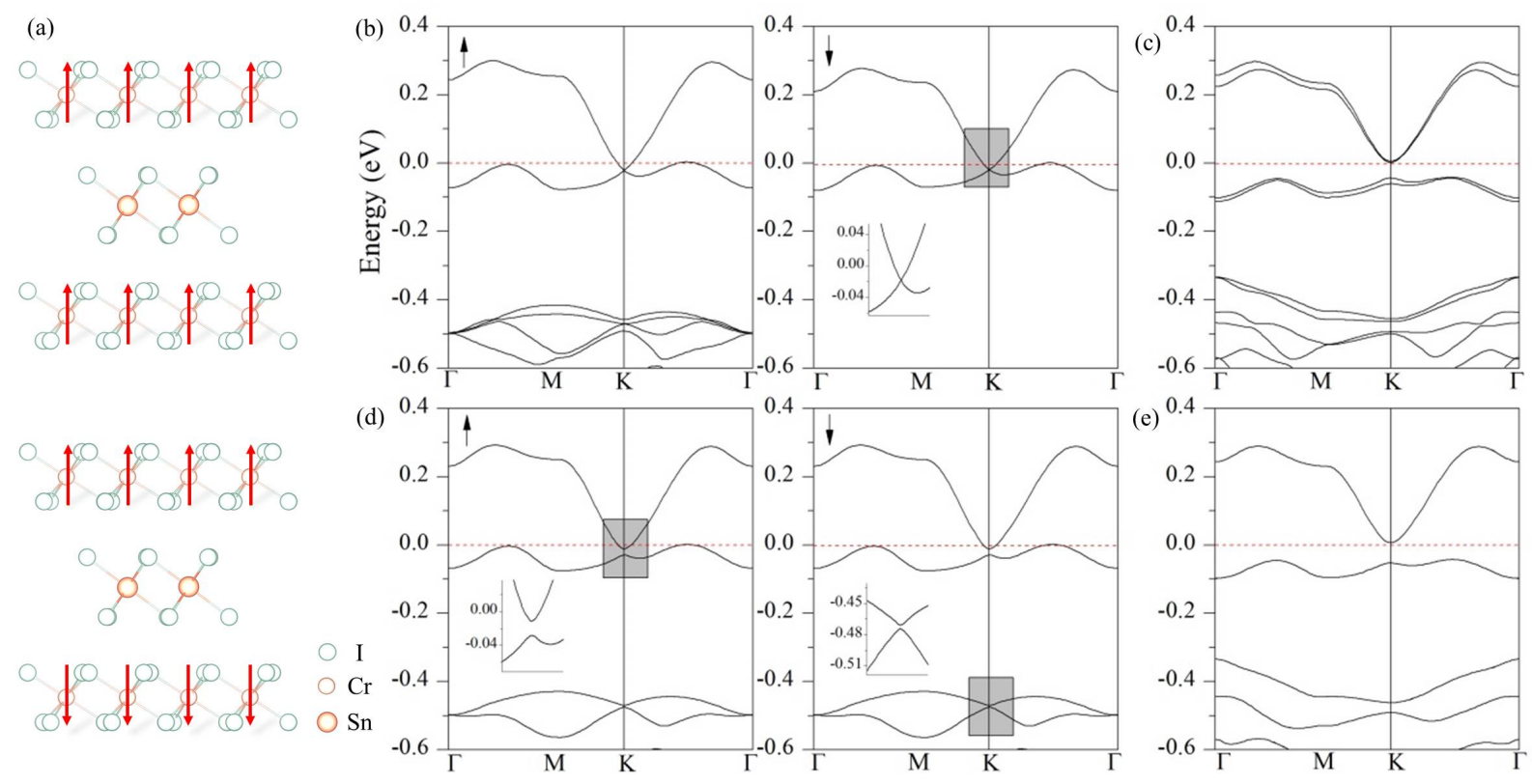

Figure 5. (a) The illustration of ferromagnetic (top) and antiferromagnetic (bottom) alignment of the $\mathrm{CrI}_{3} / \mathrm{SnI}_{3} / \mathrm{CrI}_{3}$ trilayer heterostructure. The ferromagnetic band structure without (b) and with (c) SOC. The antiferromagnetic band structure without (d) and with (e) SOC. In (b) and (d) the left and right panels correspond to spin-up and spin-down band structures, respectively. The insets show the band dispersion near the original Dirac point.

the character tables of the group of wave vector of $C_{3 i}^{1}$, it is found that there are doubly degenerate representations at $\Gamma$ and $K$. Thus the Dirac point at $K$ is preserved under ferromagnetic alignment. However, the character tables of the group of wave vectors of space group $C_{3}^{1}$ show a tiny but important difference. At $\Gamma$, there are one non-degenerate and one doubly degenerate representation, which accounts for the preservation of double degeneration at $\Gamma$ under antiferromagnetic alignment. While at $K$, there are three representations, one is non-degenerate, the other two are also non-degenerate. This explains the splitting of Dirac point at $K$. However, the last two representations at $K$ have an extra "degeneracy" with representations at $-K$, if $T$ is present. When $T$ is not present, as in our case, the examination of the magnetic space group of $C_{3 i}^{1}\left(C_{3}^{1}\right)$ shows that this "degeneracy" still exists, and we indeed find that the band structure along $\Gamma K$ is the same as that along $\Gamma(-K)$.

The effect of large SOC on magnetism is revealed by the change of the magnetic moment of the unit cell. Under ferromagnetic alignment, the $\mathrm{SnI}_{3}$ layer shows diamagnetism without SOC, and shows Van Vleck paramagnetism with SOC. This is confirmed by replacing the $\mathrm{SnI}_{3}$ layer with $\mathrm{BiI}_{3}$, under which case the magnetic moment of the unit cell is $12.0000 \mu_{B}$ (Note that there are $4 \mathrm{CrI}_{3}$ formula units per unit cell, this corresponds to $3.0000 \mu_{B}$ per $\mathrm{Cr}$ atom). This weak magnetism in $\mathrm{SnI}_{3}$ monolayer is revealed by the band structure with SOC, as shown in Figure 5(c), where the four bands near $E_{F}$ are not doubly degenerate. However, under antiferromagnetic 
Table 1. The magnetic moment per unit cell under ferromagnetic (FM) and antiferromagnetic (AFM) alignments for the heterostructure, in unit of $\mu_{B}$.

\begin{tabular}{c|cc}
\hline alignment & without SOC & with SOC \\
\hline FM & 11.9906 & $(0.0001,0,12.0932)$ \\
AFM & 0.0000 & $(0,0.0002,0)$ \\
\hline
\end{tabular}

alignment, no magnetism is introduced into the $\mathrm{SnI}_{3}$ monolayer, and the four bands near $E_{F}$ are doubly degenerate, as in Figure 5 (e). Weak paramagnetism is also seen in $\mathrm{Bi}_{2} \mathrm{Se}_{3}[14$, and is supposed to open an gap for the edge state. The DzyaloshinskiiMoriya interaction [45, 46], if exists in this system, is believed to be weak, as revealed by the tiny magnetic moment in $y$ direction under antiferromagnetic alignment.

\subsection{Magnetic proximity effect induced topological phase transition}

Under the antiferromagnetic alignment of $\mathrm{CrI}_{3}$ layers, the trilayer structure breaks both time-reversal and inversion symmetry, but preserves the symmetry of their combination $T P=i \sigma_{x} s_{y} K$. The perturbation satisfying these symmetry conditions is $H_{A F M}=$ $\mu \sigma_{z} s_{z}$. So the effective Hamiltonian including both SOC and antiferromagnetism is

$$
H_{e f f}(\vec{k})=v_{F}\left(k_{x} \sigma_{x} \tau_{z}+k_{y} \sigma_{y}\right)+\lambda \sigma_{z} \tau_{z} s_{z}+\mu \sigma_{z} s_{z}
$$

$H_{\text {eff }}$ can be block diagonalized in the eigenbasis of $s_{z}$, which means the spin-up and spin-down Hamiltonian can be separately studied. For each spin, the Hamiltonian is the same as the effective Hamiltonian of Haldane model, which includes the $T$-breaking term $\lambda \sigma_{z} \tau_{z}$ and the $P$-breaking term $\mu \sigma_{z}$. In Haldane model, the fermions are spinless, and the competition between the two terms leads to a topological phase transition between a trivial insulator and a Chern insulator. Here, since both spins are taken into account, the competition between the two terms leads to a topological phase transition between a trivial antiferromagnetic insulator and a TI. The phase transition occurs at $|\mu|=|\lambda|$. When $|\mu|>|\lambda|$, the system is a trivial insulator; when $|\mu|<|\lambda|$, it is a TI. The $\mathrm{CrI}_{3} / \mathrm{SnI}_{3} / \mathrm{CrI}_{3}$ trilayer system belongs to the latter, since the antiferromagnetism is not large enough to surpass the SOC. We can expect that in other similarly layered systems, where the SOC is smaller or the antiferromagnetism is larger, the magnetic proximity effect will induce a topological phase transition. Note that since $s_{z}$ is conserved, although time-reversal symmetry is broken, the edge states do not hybridize and are still gapless.

\section{Conclusion}

In conclusion, we have constructed a $\mathrm{CrI}_{3} / \mathrm{SnI}_{3} / \mathrm{CrI}_{3}$ trilayer heterostructure model to study the magnetic proximity effect acting on a $2 \mathrm{D} \mathrm{TI}$. The $\mathrm{SnI}_{3}$ monolayer is in $\mathrm{BiI}_{3}$ structure. The band structure shows Dirac type dispersion near the Fermi energy when SOC is ignored, and a gap (about $0.11 \mathrm{eV}$ ) opens after SOC is taken into account. 
The non-vanishing $Z_{2}$ invariant, together with the effective Hamiltonian same as KaneMele model, suggest that it is a $Z_{2}$ TI. We find that if the magnetic moments of Cr lie ferromagnetically between layers, the Dirac point is preserved; while if they lie antiferrromagnetically, a gap about $16 \mathrm{meV}$ is opened. This is explained by the fact that ferromagnetic and antiferromagnetic alignment lead to different magnetic space groups $C_{3 i}^{1}$ and $C_{3 i}^{1}\left(C_{3}^{1}\right)$. The gap opened by the magnetic proximity effect under the antiferromagnetic alignment competes with that opened by SOC, determining whether the system is a trivial antiferromagnetic insulator or a TI. Our work shows that magnetic proximity effect can tune a topological phase transition between a trivial insulator and a TI.

\section{Acknowledgments}

The authors would like to thank M. Z. Liu and Z. Yan for helpful discussions. D.Z. thanks the financial support by NSFC (No.11574403) and Guangzhou science and technology project (No.201707020002). Y.Z., L.W. and D.X.Y. are supported by the National Key R\&D Program of China 2017YFA0206203 and 2018YFA0306001, NSFC11574404, the National Supercomputer Center in Guangzhou,and the Leading Talent Program of Guangdong Special Projects.

\section{Appendix}

The first-principles calculations were carried out by using the Vienna ab initio simulation package (VASP) [47], with pseudopotentials constructed by projector augmented wave (PAW) method [48]. The generalized gradient approximation (GGA) as suggested by Perdew, Burke and Ernzerhof (PBE) [49] was used to depict the exchange and correlation interaction. A cutoff energy of $700 \mathrm{eV}$ and a Monkhorst-Pack grid[50] with $15 \times 15 \times 1$ were used to ensure convergence of $1 \mathrm{meV}$ per unit cell for the total energy. The atoms are allowed to relax until the forces were smaller than $0.01 \mathrm{eV} / \AA$. In the phonon calculation the atoms were relaxed further until the forces were smaller than $10^{-8} \mathrm{eV} / \AA$, and Phonopy [51] was used to prepare the supercell and analyze the phonon spectrum. The zero-damping DFT-D3 method of Grimme[52] was employed to describe the van der Waals interaction between the layers.

Here we give the calculation of $Z_{2}$ invariant. Due to the presence of inversion symmety, we can use $\mathrm{Fu}$ and Kane's method[53] to calculate the $Z_{2}$ invariant by computing the parity eigenvalues of occupied levels at four time-reversal invariant momenta (TRIM). The results are listed in Table 2. According to their method, the $Z_{2}$ invariant $\nu$ is defined as

$$
(-1)^{\nu}=\prod_{i} \delta_{i}=\prod_{i} \prod_{m=1}^{N} \xi_{2 m}\left(\Gamma_{i}\right)
$$

where $\Gamma_{i}$ 's are the four TRIMs in the Brillouin zone, $\xi_{2 m}\left(\Gamma_{i}\right)= \pm 1$ is the parity eigenvalue of the $2 m$-th valence band at $\Gamma_{i}$. If $\nu=1$, the system is TI, while if $\nu=0$, 
the system is a trivial insulator. At the end of Table 2 we give the sign of $\delta_{i}$. Since $\delta=1$ at the three $M$ 's and $\delta=-1$ at $\Gamma$, we have $\nu=1$, confirming the $\mathrm{SnI}_{3}$ monolayer is a TI.

Table 2. The parity eigenvalues of the $2 m$-th valence band at four TRIMs: $\Gamma(0,0,0), M_{1}(1 / 2,0,0), M_{2}(0,1 / 2,0)$ and $M_{3}(1 / 2,-1 / 2,0)$. The last second column is the parity of the bottom conduction band, and the last column is the value of $\delta$.

\begin{tabular}{c|ccccccccccccccc}
\hline $2 \mathrm{~m}$ & 2 & 4 & 6 & 8 & 10 & 12 & 14 & 16 & 18 & 20 & 22 & 24 & 26 & 28 & 30 \\
\hline$M$ & +1 & -1 & -1 & +1 & +1 & -1 & +1 & -1 & -1 & +1 & -1 & +1 & -1 & +1 & -1 \\
$\Gamma$ & -1 & +1 & +1 & -1 & -1 & -1 & +1 & +1 & +1 & -1 & +1 & +1 & +1 & -1 & -1 \\
\hline $2 \mathrm{~m}$ & 32 & 34 & 36 & 38 & 40 & 42 & 44 & 46 & 48 & 50 & 52 & 54 & 56 & 58 & 60 \\
\hline$M$ & +1 & +1 & -1 & -1 & +1 & -1 & +1 & -1 & +1 & -1 & +1 & -1 & +1 & -1 & +1 \\
$\Gamma$ & -1 & -1 & +1 & +1 & +1 & -1 & +1 & -1 & +1 & -1 & +1 & -1 & +1 & +1 & -1 \\
\hline $2 \mathrm{~m}$ & 62 & 64 & 66 & 68 & 70 & 72 & $\delta_{i}$ & & & & & & & & \\
\hline$M$ & -1 & +1 & -1 & +1 & -1 & +1 & +1 & & & & & & & & \\
$\Gamma$ & -1 & +1 & -1 & -1 & +1 & -1 & -1 & & & & & & & & \\
\hline
\end{tabular}

\section{References}

[1] Kane C L and Mele E J 2005 Phys. Rev. Lett. 95(14) 146802

[2] Hasan M Z and Kane C L 2010 Rev. Mod. Phys. 82(4) 3045

[3] Kane C L and Mele E J 2005 Phys. Rev. Lett. 95(22) 226801

[4] Bernevig B A, Hughes T L and Zhang S C 2006 Science 3141757

[5] Ando Y 2013 J.Phys. Soc. Jpn. 82102001

[6] Klimovskikh I I, Otrokov M M, Voroshnin V Y, Sostina D, Petaccia L, Di Santo G, Thakur S, Chulkov E V and Shikin A M 2017 ACS Nano 11368

[7] Avsar A, Tan J Y, Taychatanapat T, Balakrishnan J, Koon G K W, Yeo Y, Lahiri J, Carvalho A, Rodin A S, OFarrell E C T, Eda G, Castro Neto A H and zyilmaz B 2014 Nat. Commun. 5 4875

[8] Wang Z, Ki D, Chen H, Berger H, MacDonald A H and Morpurgo A F 2015 Nat. Commun. 6 8339

[9] Liu C C, Feng W and Yao Y 2011 Phys. Rev. Lett. 107(7) 076802

[10] Xu Y, Yan B, Zhang H J, Wang J, Xu G, Tang P, Duan W and Zhang S C 2013 Phys. Rev. Lett. 111(13) 136804

[11] Wada M, Murakami S, Freimuth F and Bihlmayer G 2011 Phys. Rev. B 83(12) 121310

[12] Qian X, Liu J, Fu L and Li J 2014 Science 3461344

[13] Tokura Y, Yasuda K and Tsukazaki A 2019 Nat. Rev. Phys. 1126

[14] Yu R, Zhang W, Zhang H J, Zhang S C, Dai X and Fang Z 2010 Science 32961

[15] Nomura K and Nagaosa N 2011 Phys. Rev. Lett. 106(16) 166802

[16] Chen Y L, Chu J H, Analytis J G, Liu Z K, Igarashi K, Kuo H H, Qi X L, Mo S K, Moore R G, Lu D H, Hashimoto M, Sasagawa T, Zhang S C, Fisher I R, Hussain Z and Shen Z X 2010 Science 329659

[17] Lee I, Kim C K, Lee J, Billinge S J L, Zhong R, Schneeloch J A, Liu T, Valla T, Tranquada J M, Gu G and Davis J C S 2015 Proc. Natl. Acad. Sci. USA 1121316

[18] Lee C, Katmis F, Jarillo-Herrero P, Moodera J S and Gedik N 2016 Nat. Commun. 712014

[19] Katmis F, Lauter V, Nogueira F S, Assaf B A, Jamer M E, Wei P, Satpati B, Freeland J W, Eremin I, Heiman D, Jarillo-Herrero P and Moodera J S 2016 Nature 533513 
Topological phase transition induced by magnetic proximity effect in two dimensions 11

[20] McGuire M A, Dixit H, Cooper V R and Sales B C 2015 Chem. Mater. 27612

[21] Huang B, Clark G, Navarro-Moratalla E, Klein D R, Cheng R, Seyler K L, Zhong D, Schmidgall E, McGuire M A, Cobden D H, Yao W, Xiao D, Jarillo-Herrero P and Xu X 2017 Nature 546 270

[22] Huang B, Clark G, Klein D R, MacNeill D, Navarro-Moratalla E, Seyler K L, Wilson N, McGuire M A, Cobden D H, Xiao D, Yao W, Jarillo-Herrero P and Xu X 2018 Nat. Nanotech. 13544

[23] Haldane F D 1988 Phys. Rev. Lett. 612015

[24] Edelstein V 1990 Solid State Commun. 73233

[25] Wang Y, Zhu D, Wu Y, Yang Y, Yu J, Ramaswamy R, Mishra R, Shi S, Elyasi M, Teo K L, Wu $\mathrm{Y}$ and Yang H 2017 Nat. Commun. 81364

[26] Mellnik A R, Lee J S, Richardella A, Grab J L, Mintun P J, Fischer M H, Vaezi A, Manchon A, Kim E A, Samarth N and Ralph D C 2014 Nature 511449

[27] Han J, Richardella A, Siddiqui S A, Finley J, Samarth N and Liu L 2017 Phys. Rev. Lett. 119(7) 077702

[28] Podraza N J, Qiu W, Hinojosa B B, Xu H, Motyka M A, Phillpot S R, Baciak J E, TrolierMcKinstry S and Nino J C 2013 J. Appl. Phys. 114033110

[29] Lehner A J, Wang H, Fabini D H, Liman C D, Hébert C A, Perry E E, Wang M, Bazan G C, Chabinyc M L and Seshadri R 2015 Appl. Phys. Lett. 107131109

[30] Nason D and Keller L 1995 J. CRYST GROWTH 156221

[31] Slater J C 1965 Quantum Theory of Molecules and Solids, Vol.2: Symmetry and Energy Bands in Crystals (New York: McGraw-Hill)

[32] Herring C 1937 Phys. Rev. 52(4) 361

[33] Castro Neto A H, Guinea F, Peres N M R, Novoselov K S and Geim A K 2009 Rev. Mod. Phys. 81(1) 109

[34] Yao Y, Ye F, Qi X L, Zhang S C and Fang Z 2007 Phys. Rev. B 75(4) 041401

[35] Min H, Hill J E, Sinitsyn N A, Sahu B R, Kleinman L and MacDonald A H 2006 Phys. Rev. B 74(16) 165310

[36] Downing C A and Portnoi M E 2017 J.Phys.: Condens. Matter 29315301

[37] Webster L and Yan J A 2018 Phys. Rev. B 98(14) 144411

[38] Zhong D, Seyler K L, Linpeng X, Cheng R, Sivadas N, Huang B, Schmidgall E, Taniguchi T, Watanabe K, McGuire M A, Yao W, Xiao D, Fu K M C and Xu X 2017 Sci. Adv. 3 e1603113

[39] Ge M, Su Y, Wang H, Yang G and Zhang J 2019 RSC Adv. 914766

[40] Zhang P, Peng X L, Qian T, Richard P, Shi X, Ma J Z, Fu B B, Guo Y L, Han Z Q, Wang S C, Wang L L, Xue Q K, Hu J P, Sun Y J and Ding H 2016 Phys. Rev. B 94104510

[41] Bertolazzi S, Brivio J and Kis A 2011 ACS Nano 59703

[42] Slater J C 1951 Phys. Rev. 82538

[43] Bradley C and Cracknell A 1972 The mathematical theory of symmetry in solids : representation theory for point groups and space groups (Oxford : Clarendon Press)

[44] Dimmock J O and Wheeler R G 1962 Phys. Rev. 127(2) 391

[45] Dzyaloshinsky I 1958 J. Phys. Chem. Solids 4241

[46] Moriya T 1960 Phys. Rev. 120(1) 91

[47] Kresse G and Furthmüller J 1996 Phys. Rev. B 54(16) 11169

[48] Blöchl P E 1994 Phys. Rev. B 50(24) 17953

[49] Perdew J P, Burke K and Ernzerhof M 1996 Phys. Rev. Lett. 77(18) 3865

[50] Monkhorst H J and Pack J D 1976 Phys. Rev. B 13(12) 5188

[51] Togo A and Tanaka I 2015 Scripta Materialia 1081

[52] Grimme S, Antony J, Ehrlich S and Krieg H 2010 J. Chem. Phys. 132154104

[53] Fu L and Kane C L 2007 Phys. Rev. B 76(4) 045302 